\title{
Linewidth Pressure Measurement: A New Technique for High Vacuum Characterization
}

\author{
Chad A. Jones, David V. Dearden
}

Department of Chemistry and Biochemistry, Brigham Young University, C100 Benson Science Building, Provo, Utah 846025700, USA

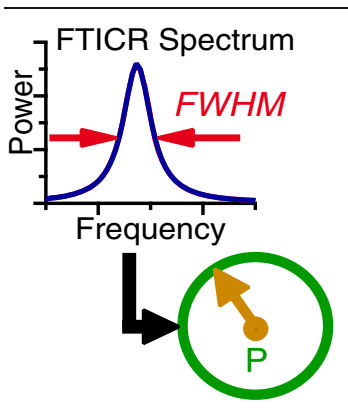

Abstract. Pressure measurement is often the limiting factor in the accuracy of
quantitative ion-molecule experiments. We present a new method for pressure
measurement based on analysis of pressure-limited Fourier transform ion cyclotron
resonance (FTICR) linewidths for well-characterized collisions of $\mathrm{Ar}^{+}$with Ar. The
kinetic energy dependence of $\mathrm{Ar}^{+} / \mathrm{Ar}$ collision cross sections is well-described using
a single-parameter fitting procedure, which results in pressure measurements in
good agreement with those from a cold cathode tube and from measurement of total
ion signal following electron impact ionization. The new method avoids problems
inherent in ionization-based methods, such as those arising from differences in
ionization potential or perturbations to the pressure that occur during electron ionization of the gas to be measured, and should be applicable in the trapping cells of FTICR and Orbitrap mass spectrometers.

Keywords: Fourier transform ion cyclotron resonance mass spectrometry, Linewidth, Pressure measurement, Collision cross section

Received: 27 August 2014/Revised: 25 September 2014/Accepted: 22 October 2014/Published Online: 12 November 2014

\section{Introduction}

G as pressure plays a fundamental role in many important chemical processes, including the establishment of physical and chemical equilibria and the determination of rates of chemical reactions. Accurate measurement of pressure is essential for characterizing such processes, and pressure measurement is often the limiting factor in many quantitative experiments [1].

Specifically, in Fourier transform ion cyclotron resonance (FTICR) mass spectrometric measurements of reaction rates [2, 3], collisional dissociation thresholds [4-8], or collision cross sections [9], accurate pressure determinations in the ion trapping cell are essential. In the relevant pressure range (typically $10^{-10}$ to $10^{-5} \mathrm{mbar}$ ), pressures are almost always measured using ionization-based techniques [10]. Most commonly, these involve either a Bayard-Alpert style hot cathode ionization gauge [11] or a Penning-style cold cathode gauge [12, 13].

Electronic supplementary material The online version of this article (doi:10.1007/s13361-014-1031-8) contains supplementary material, which is available to authorized users.

Correspondence to: David V. Dearden; e-mail: david_dearden@byu.edu
All ionization-based methods, including these, fundamentally involve ionization of the neutral gas and measurement of the resulting ion current [10]. The ion current is proportional to the neutral gas pressure and also depends on the ionization cross section of the gas.

However, the high magnetic field required for FTICR experiments presents some special challenges because ion motion and the resulting ion current measurement are strongly affected by the magnetic field. Most commonly, ionization gauge transducers on FTICR instruments are placed outside the high-field region of the magnet, sometimes as much as a meter or more away from the ion trapping cell. Gas conductance between the transducer and the trapping cell can result in pressure differences between the transducer and trapping cell of as much as an order of magnitude [14]. When pressure measurements are critical, corrections for this difference must be made, typically by measuring the rate of a well-characterized ion-molecule reaction in the trapping cell and using the known rate constant to determine the actual pressure in the trapping cell [14].

In addition to the problems inherent to operating in a high magnetic field, ionization-based methods suffer other problems [10]. The transducers act as low-speed ion pumps, so their operation may perturb the measurement. Ionization techniques are influenced by deposition of material on the surfaces of the 
transducer, which may change with time. Hot cathode devices require bakeout and thermal equilibration, and outgassing from the heated filament may additionally perturb the pressure. In addition, in cold cathode devices, at low pressures it can be difficult to initiate the plasma that is required to ionize the neutral gas, and small nonlinear discontinuities between pressure and ion current have been reported [15]. As a result, the sensitivity of an ionization-based measurement may vary by as much as a factor of two to three over a period of several days.

We recently introduced a new method of measuring collision cross sections using an FTICR instrument (cross sectional areas from analysis of Fourier transform ion cyclotron resonance, or "CRAFTI") [9, 16]. In FTICR experiments, ions are detected by using an applied rf pulse to excite them into phase coherent cyclotron motion, and then detecting the resulting image current induced by the coherently orbiting ions. CRAFTI measurements are based on the dependence of full width at half maximum (FWHM) FTICR linewidths on collisions that dephase the coherently orbiting ions (under pressurelimited conditions), and depend inversely on the neutral collision gas number density $\left(n_{n}\right)$, as shown in $(1)[9,16]$.

$\sigma=\frac{F W H M}{n_{n}} \frac{\left(m_{\text {ion }}+M_{\text {neutral }}\right)}{M_{\text {neutral }}} \frac{m_{\text {ion }}}{q} \frac{d}{\beta V_{p p} t_{\text {exc }}}$

Here, $\sigma$ is the dephasing cross section, $m_{\text {ion }}$ and $M_{\text {neutral }}$ are the masses of the ion and neutral, respectively, $q$ is the ion charge, $d$ is the cell diameter, $\beta$ is the cell geometry factor, $V_{p p}$ is the peak-to-peak excitation voltage, and $t_{e x c}$ is the duration of the single-frequency resonant excitation event used to induce the initial phase coherence.

It is easy to see that (1) can be solved for neutral number density, and that (all other things being equal) number density is directly proportional to the FWHM linewidth. All the terms in the resulting equation except $\sigma$ are either known or easily measured. This suggests that for systems where $\sigma$ is known and the linewidth is pressure-limited, FWHM FTICR linewidth measurements can be used to determine absolute pressures. This is an attractive idea because it might enable measurement of pressures directly in the FTICR cell, without depending on ionization potential or requiring an ion current measurement. A similar approach that used Barker-Ridge theory [17] to estimate momentum transfer cross sections and determined pressures in the trapping cell from the rate of transient decay in a conventional ion cyclotron resonance mass spectrometer has been described previously [18]. However, that treatment assumed ion-molecule collisions at thermal energies, which will introduce some error because the ions comprising a coherently orbiting packet are always at kinetic energies much greater than thermal, and cross sections are kinetic energy-dependent [19].

For the approach we propose, ions with well-characterized (preferably via experiment) collision cross sections are needed. Both ion mobility spectrometry (IMS) [20-22] and travelingwave ion mobility spectrometry (TWIMS) [23, 24] have generated large databases of measured ion-neutral collision cross sections. However, identification of suitable ions with known cross sections is complicated by the fact that ion-neutral cross sections are dependent on the kinetic energy of the collision and on the neutral collision gas. Therefore, directly comparing CRAFTI cross sections to IMS or TWIMS is not straightforward because the experimental conditions for these techniques are quite different from those that pertain to the FTICR cell when using CRAFTI. For example, while both IMS and TWIMS involve many low energy (thermal) collisions (usually with $\mathrm{He}$ or $\mathrm{N}_{2}$ ), CRAFTI measurements involve a single high energy $(\sim 1-10 \mathrm{keV})$ collision (usually with Ar or some other heavier collision partner). This problem is further complicated by the fact that all dephasing collisions, including collisions that lead to dissociation, play a role in determining CRAFTI cross sections, whereas ion mobility measurements are dominated by momentum transfer collisions. As a result, CRAFTI cross sections are typically larger than measured IMS, TWIMS, or computed cross sections that are designed to model IMS or TWIMS. This makes cross sections measured using mobility methods difficult to use for our purposes.

Fortunately, a suitable set of kinetic energy-resolved cross sections is available for collisions of $\mathrm{Ar}^{+}$with neutral $\mathrm{Ar}$ [19]. This is convenient because our primary application is pressure measurements in support of CRAFTI cross section determinations, and Ar is our collision gas of choice [16]. The purpose of this paper is to demonstrate this new method of measuring pressures that does not rely on ion current measurements, specifically to show that FTICR linewidth measurements can be used to accurately measure absolute pressures in the trapping cells of FTICR instruments. We term the new method linewidth pressure measurement, and refer to it herein by the acronym "LIPS."

\section{Experimental}

\section{Materials}

Ar gas (99.95\%) was purchased from Airgas (Radnor, PA, USA).

\section{Instrumentation}

All experiments were performed using a Bruker APEX 47e Fourier transform ion cyclotron resonance mass spectrometer with an Infinity trapping cell [25]. The instrument was controlled using a MIDAS Predator data system (National High Magnetic Field Laboratory; Tallahassee, FL) [26, 27]. Radio frequency (rf) excitation amplitudes were measured using an oscilloscope at the output of the final excitation amplifier.

\section{Pressure Control}

Because pressure control via a Freiser-type pulsed leak valve [28] is crucial to our experiments, we describe the operation of the pulsed leak valve in detail. The pulsed leak valve on our instrument consists of a 0.004 in. orifice pressurization 
solenoid valve backed by a 28 psig Ar supply line; and a 0.039 in. orifice evacuation solenoid valve connected to a mechanical vacuum pump (both valves from General Valve Corp.; Fairfield, NJ, USA). Both solenoid valves were connected to the high-pressure side of a precision variable leak valve (Varian, Palo Alto, CA, USA) set at a constant value.

When no leak is desired, the evacuation solenoid valve is left open and the pressurization solenoid valve is left closed so that the backing pressure on the precision leak valve remains low (typically at the base pressure of the mechanical pump, about $\left.1 \times 10^{-3} \mathrm{mbar}\right)$. This makes the leak rate through the leak valve low, leaving the trapping cell essentially at its baseline pressure (about $5 \times 10^{-9} \mathrm{mbar}$ ). To increase the steady-state pressure in the trapping cell, the evacuation solenoid valve is closed and the pressurization solenoid valve is momentarily opened. This increases the backing pressure on the precision leak valve so that the pressure in the trapping cell rises from baseline to a constant value that is determined by the leak rate. The leak rate depends on how much gas is allowed into the volume on the high pressure side of the leak valve. This amount of gas is controlled by the computer-controlled duration of the pressurization pulse. The steady-state pressures attained in the trapping cell when the pulsed leak is operated range from about $1 \times 10^{-8} \mathrm{mbar}$ (for a $5 \mathrm{~ms}$ pressurization pulse) up to about $5 \times$ $10^{-4}$ mbar (after leaving the pressurization solenoid open $500 \mathrm{~ms}$ or more). We therefore varied the steady-state pressures obtained with the pulsed leak system by varying the length of time the pressurization solenoid was pulsed open.

The rise time from pressurization solenoid activation to attainment of steady-state pressure is approximately $500 \mathrm{~ms}$ (vide infra). Thereafter, the pressure remains constant for at least tens of seconds (much longer than was used in any of the experiments reported here) or until the evacuation solenoid valve is opened. Opening the evacuation solenoid reduces the backing pressure behind the leak valve so the leak rate falls until the pressure returns to baseline. The time required to return completely to baseline pressure after opening the evacuation valve in our system is approximately $2 \mathrm{~s}$, although most of the pressure drop occurs in the first $500 \mathrm{~ms}$.

\section{Procedures}

Conventional pressure measurements were performed using a cold cathode gauge (Balzers, Fürstentum, Lichtenstein) mounted outside the high field region of the instrument, about $1 \mathrm{~m}$ from the trapping cell and immediately adjacent to the pulsed leak valve. All pressures were adjusted for the ionization cross section of neutral $\operatorname{Ar}[1]$.

In most of our LIPS experiments, $\mathrm{Ar}^{+}$ions were created by first pulsing neutral Ar into the trapping cell to about $5 \times 10^{-7}$ mbar and ionizing with a $50 \mathrm{~ms}, 100 \mathrm{eV}$ pulse from an electron gun mounted on the trapping cell axis. Following ionization, the pulsed leak was evacuated and the system was pumped for $500 \mathrm{~ms}$ to remove most of the neutral Ar and return the system close to baseline pressure.

For experiments measuring the time dependence of pressures in the trapping cell following pulsed leak valve pressurization, which required better time resolution, a shorter $5 \mathrm{~ms}, 100 \mathrm{eV}$ pulse from the electron gun was used to produce ions. Because the total FTICR experimental sequence was long enough to allow reactive collisions (mainly charge transfer to form $\mathrm{H}_{2} \mathrm{O}^{+}$) at the pressures employed, the total ion intensity of all species was measured as a function of the steady-state Ar pressure achieved at various pulsed valve pressurization durations. A calibration curve was generated to relate total ion intensity to steady-state Ar pressure as determined using the cold cathode tube.

A typical LIPS experiment was done by first creating $\mathrm{Ar}^{+}$as described above. Next, neutral Ar was leaked into the system using various pulsed solenoid valve pressurization durations to produce different steady state pressures. After a $500 \mathrm{~ms}$ delay to allow the pressure to attain its steady state value, the $\mathrm{Ar}^{+}$ions were then coherently excited using a single-frequency resonant $\mathrm{rf}$ pulse, and the resulting transient signal was digitized using $64 \mathrm{k}$ data points at a bandwidth of $2.63 \mathrm{MHz}$. This results in digitizing for $12.45 \mathrm{~ms}$, whereas the coherent signal decays to baseline noise levels after approximately $8 \mathrm{~ms}$ at the lowest pressure used. This ensures that the signal transients were not truncated by the detection duration. The power spectrum $\mathrm{Ar}^{+}$full width at half maximum linewidth was computed for each transient using the Igor Pro software package (ver. 6, Wavemetrics; Lake Oswego, OR, USA) [9]. (1), using the appropriate cross section for the experimental $\mathrm{Ar}^{+}$kinetic energy, yields a measured pressure corresponding to the steady state pressure produced by that particular pulsed leak pressurization duration. Because the steady state pressures created by the pulsed leak valve were very reproducible [28], in practice the pulsed leak duration could be used thereafter to generate known steady-state pressures.

\section{Results and Discussion}

\section{LIPS Pressure Calibration from Fitting $\mathrm{Ar}^{+}$in $\mathrm{Ar}$ Collision Cross Sections}

CRAFTI cross sections for collisions of $\mathrm{Ar}^{+}$with $\mathrm{Ar}$ at a particular lab frame kinetic energy $K E_{\text {lab }}$ (from (2)) [29] were measured according to (1) by measuring FWHM $\mathrm{Ar}^{+}$ linewidths over a range of $\mathrm{Ar}$ pressures. These pressures were obtained by varying the duration of the pulsed leak valve pressurization pulse, with a constant backing pressure behind the pressurization solenoid valve. We assume that the steadystate pressure achieved with the pulsed leak valve is linearly proportional to the time that the high-pressure solenoid valve is opened (please see Supplementary Material); justification for this assumption is provided below.

$$
K E_{\text {lab }}=\frac{1.2067 \times 10^{7} \beta^{2} q^{2} V_{p p}^{2} t_{e x c}^{2}}{d^{2} m_{\text {ion }}}
$$

Here all symbols are the same as defined for (1).

As was noted above, all the terms in (1) except the neutral number density, $n_{n}$, are known. The CRAFTI cross sections 
measured as described above were compared with cross sections from the literature [19] for the same lab frame kinetic energies, and the square of the difference was calculated. We then minimized the sum of squares of these differences for a range of kinetic energies by varying the proportionality constant that converts the pulsed leak duration to $n_{n}$ as the only free parameter in the fit.

The results of our fitting procedure are shown in Figure 1. The fitted cross sections agree well with those from the literature [19] over a range of more the $10 \mathrm{keV}$ in lab frame kinetic energies; these energies correspond to those typically needed to produce well-tuned FTICR spectra. The quality of this fit provides some justification of our assumption of linearity between pulsed leak duration and pressure.

It is also noteworthy that for the $\mathrm{Ar}^{+}$in $\mathrm{Ar}$ system, cross sections decrease with increasing kinetic energy as expected from collision theory. This is in contrast to the CRAFTI cross sections for larger molecular ions we reported earlier $[9,16]$, which generally increase with increasing kinetic energy. We believe the increase occurs because of increasing contributions to the CRAFTI cross section from collision-induced dissociation as kinetic energy increases. For monoatomic $\mathrm{Ar}^{+}$, collision-induced dissociation is not possible and the cross sections decrease with kinetic energy as expected. This phenomenon will be the subject of a future report.

\section{Comparison of LIPS and Cold Cathode Tube Absolute Pressures}

The pressure calibration described above enables measurement of absolute pressures through measuring $\mathrm{Ar}^{+}$in $\mathrm{Ar}$ pressurelimited linewidths. The results of a set of measurements as the pulsed leak pressurization solenoid activation duration is varied are shown in Figure 2, along with steady state pressures obtained from the cold cathode tube, uncorrected for transmission between the trapping cell and pressure transducer.

The absolute steady state pressures via the two methods agree within a factor of two throughout the range examined,

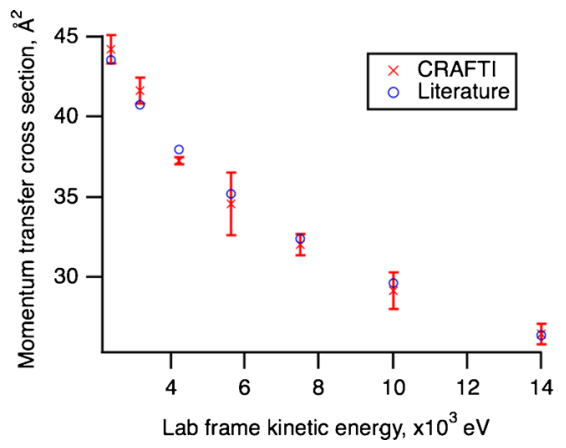

Figure 1. Momentum transfer cross sections for $\mathrm{Ar}^{+}$in $\mathrm{Ar}$ as a function of lab frame kinetic energy, from the literature (Reference [19]) and from CRAFTI measurements least-squares fitted to the literature values with the pulsed leak valve-to-pressure conversion factor as the only free parameter. Error bars represent \pm 1 standard deviation from the procedure used to calculate cross section

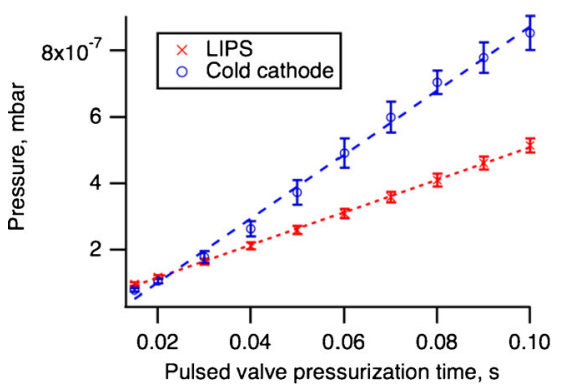

Figure 2. Absolute steady state pressures as a function of pulsed leak valve pressurization solenoid duration, measured using linewidth pressure measurement (LIPS) calibrated using $\mathrm{Ar}^{+}$in $\mathrm{Ar}$ collision cross sections, compared with steady state pressures determined using an uncorrected cold cathode tube. Error bars represent \pm 1 standard deviation over repeated measurements, and dotted lines are linear fits to the data

although pressures from the cold cathode tube are generally greater than those from LIPS, as expected given the location of the cold cathode transducer proximate to the pulsed leak inlet, with the ion trapping cell about $1 \mathrm{~m}$ away. We note that absolute agreement between the two methods is not expected because the cold cathode tube measures the pressure outside the high magnetic field region of the FTICR, whereas LIPS is sensitive to pressures in the trapping cell.

Both pressure measurement techniques compared in Figure 2 suggest an approximately linear relationship between pulsed leak pressurization duration and the steady state pressure achieved by the pulsed leak, again justifying our assumption of linearity (and please note that no assumption was involved in the cold cathode tube measurements). However, the linearity in the case of LIPS is somewhat better $\left(R^{2}=\right.$ 0.9994 for LIPS versus 0.9951 for the cold cathode tube). The deviations from linearity seen with the cold cathode tube are consistent with previously observed small discontinuities in the cold cathode current versus pressure relationship [15].

\section{Comparison of LIPS with Total Ion Intensities}

One of the difficulties in attempting to compare LIPS pressures with pressures measured using other techniques is that most other techniques (such as the cold cathode tube used here) do not directly measure the pressure in the trapping cell, but measure it at some remote location in the vacuum chamber. We, therefore, sought another method for comparing pressures more directly in the ion trapping region.

Signal intensity in FTICR is proportional to the number of charges held in the ion trapping cell [29]. Further, as long as the space charge limit is not approached, the number of ions produced by an electron beam pulse through the trapping cell should be proportional to the number of neutral molecules irradiated by the electron beam, which is in turn proportional to the neutral pressure. This suggests that total FTICR signal intensity following a fixed-length, constant energy pulse of electrons through the trapping cell should be a viable means of measuring the neutral pressure in the trapping cell. In fact, 
this approach was taken by Jiao et al. [28] to characterize the rise times for different pulsed leak valve configurations.

We used total FTICR signal intensity following a $5 \mathrm{~ms}$ electron beam event as a means of probing the total neutral pressure in our trapping cell, for comparison with pressures measured using LIPS. Steady-state pressure measurements using this method after various delay times following pulsedleak pressurization of the instrument showed that total signal intensity increased linearly with pulsed leak pressurization duration, indicating these experiments were conducted below the space charge limit, and again suggesting that absolute steady state pressure and pulsed leak pressurization solenoid duration are linearly related. Steady-state pressures read from the cold cathode tube were measured and used to generate a calibration curve relating total FTICR signal to cold cathode pressures. Thus, measurement of total FTICR signal intensity yields another method for determining absolute pressures, recognizing that the absolute pressures obtained from this calibration contain systematic error due to calibration with the cold cathode tube and its location remote from the trapping cell. Not surprisingly, pressures measured in this way agree within about a factor of two with LIPS pressures.

However, additional verification of the LIPS technique can be obtained from these experiments by measurement of the pressure in the ion trapping cell as a function of time after the pulsed leak valve is activated. These experiments focus on measuring the time dependence of the rising pressure immediately after the pulsed leak is activated rather than on measuring the absolute pressures. Because ions are made only during the $5 \mathrm{~ms}$ electron beam pulse, measurement of total FTICR signal offers a means of measuring pressures with higher time resolution (down to the duration of the electron beam pulse, in principle) than is possible with the cold cathode tube and its associated electronics. Similarly, LIPS measurements can be made rapidly (as long as the signal transient is comparable to the desired time resolution) by examining the rate of transient signal decay for the same group of $\mathrm{Ar}^{+}$ions. Both types of measurements reflect pressure changes within the ion trapping cell and should show changes occurring at the same rate if both accurately measure pressure as a function of time.

A comparison of the two techniques, measuring pressures by activating the electron gun at various time delays following activation of the pulsed leak via pulsing the pressurization solenoid open for $100 \mathrm{~ms}$, is shown in Figure 3 (please see Supplementary Material for uncalibrated total ion signal data). According to both techniques, the pressure in the cell rises exponentially with time to a constant, steady state value. Within experimental error, both FTICR signal intensity and LIPS give the same rise time constant (about $160 \mathrm{~ms}$ ), suggesting both methods measure the same time profile for pressure in the trapping cell following introduction of Ar through the pulsed leak. Interestingly, the standard deviation in the rise time constant via either method is around $5 \mathrm{~ms}$, which is comparable to the length of the ionization event used to create the ions. The length of the ionization event ultimately limits the time resolution of both methods.

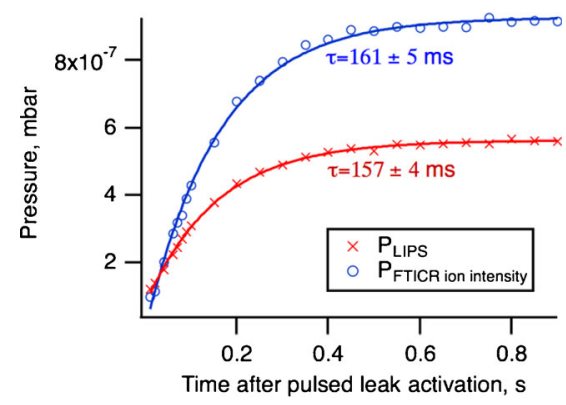

Figure 3. Variation of pressure in the FTICR trapping cell with time after pressurization of the pulsed leak valve for $100 \mathrm{~ms}$, measured using FTICR signal intensity and measured using linewidth pressure measurement (LIPS). The curves are exponential fits to the experimental data, and time constants from the fits (with standard deviations) are inset. The two methods yield the same rise time constant, although the absolute pressures differ because of systematic error in the ion intensity technique

\section{Strengths of LIPS Relative to Conventional High Vacuum Pressure Measurements}

LIPS offers several advantages in comparison with traditional methods of characterizing high vacuum. For our applications, a chief advantage is that because the ion trapping cell is the transducer, LIPS measures pressure in the cell, exactly where we want to make the measurement. Thus, LIPS techniques avoid the need to measure the pressure at some remote point and then correct back to cell pressure, potentially yielding more accurate results for experiments, such as CRAFTI cross section measurements [9], where pressure plays a crucial role.

Other advantages derive from the fact that LIPS measurements do not depend on the ionization cross sections of the gases whose pressures are measured. Although LIPS does require ions, the ions need not be made by electron impact, so perturbation of the pressure arising from the presence of a heated filament can be avoided. Therefore, there is no need for bakeout of a gauge tube or for thermal equilibration of the system, there are no ion pumping effects, and there is no outgassing from a hot filament. Similarly, there is no need to ignite a plasma.

Because electrons are not required to make a LIPS measurement, the production of soft X-rays that occurs when electrons collide with surfaces, and the corresponding residual current [10], is not a limiting factor for LIPS as it is for ionizationbased pressure measurement. Finally (and somewhat subjectively), relative to the cold cathode technique we use extensively, LIPS measurements in our hands have better reproducibility, and less day-to-day drift.

\section{Weaknesses of LIPS}

In comparison with conventional techniques, the greatest current weakness of LIPS is its limited applicability; the technique is only appropriate for instruments that generate a transient signal (from coherently-moving ions) that has a pressurelimited lifetime. Thus, LIPS is demonstrated here to work for 
FTICR instruments and will likely work in Orbitrap mass spectrometers [30], but currently is not applicable elsewhere.

LIPS measurements depend on the collision cross section of ion-neutral collisions, which are kinetic energy-dependent. Because few kinetic energy-resolved ion-neutral collision cross sections in the energy range appropriate for FTICR measurements are available, currently only a few options are available for calibrating LIPS. However, CRAFTI techniques $[9,16]$ offer a means of measuring collision cross sections in gases other than Ar, as well as their kinetic energy dependence in the appropriate energy range, so that more options should soon become available. In addition, Barker-Ridge theory [17] could be used to estimate cross sections for other combinations of ions and neutral collision gases, making LIPS measurements possible for a wider range of systems, with the caveat that some error will be introduced because the kinetic energies of the ions in these experiments are not thermal.

The time resolution of LIPS measurements is limited by the length of the required excite/detect event, which is in turn limited by the requirement that the decaying transient signal not be transform-length-limited. Because the transient decay rate is pressure-dependent, the time required to adequately characterize the linewidth is also pressure-dependent, so the time resolution of LIPS will get worse at lower pressures.

Finally, factors other than pressure certainly limit FTICR linewidths at low pressures [29] and will likely determine the low-pressure limit of the LIPS technique, which is currently not known. Because pressure and cross section are inversely proportional (1), the use of larger ions for LIPS may offset these problems and improve the low-pressure limit of the technique.

\section{Conclusions}

Measurement of FTICR linewidth for species with known collision cross sections yields absolute pressure measurements in the FTICR trapping cell that are in reasonable agreement with conventional ionization-based techniques but avoid problems arising from thermionic production of ions and a remotely-located pressure transducer. LIPS therefore represents a fundamentally new method for measuring low pressures, which should enable more accurate results for other ion chemistry experiments that are pressure-dependent and can be performed in FTICR or Orbitrap instruments. In particular, pressures determined from the LIPS technique can be used to make absolute CRAFTI cross section measurements, whereas until now only relative measurements have been possible. This may ultimately turn out to be the most important application of the LIPS method. As more absolute cross sections are accurately measured, this will, in turn, make available more possibilities for ions that can be used in LIPS.

\section{Acknowledgments}

The authors are grateful for support of this work by the US National Science Foundation under grant number CHE1412289.

\section{References}

1. Bartmess, J.E., Georgiadis, R.M.: Empirical methods for determination of ionization gauge relative sensitivities for different gases. Vacuum 33, 149153 (1983)

2. Dearden, D.V., Zhang, H., Chu, I.-H., Wong, P., Chen, Q.: Macrocyclic chemistry without solvents: gas phase reaction rates. Pure Appl. Chem. 65, 423-428 (1993)

3. Chu, I.H., Zhang, H., Dearden, D.V.: Macrocyclic chemistry in the gas phase: intrinsic cation affinities and complexation rates for alkali metal cation complexes of crown ethers and glymes. J. Am. Chem. Soc. 115, 5736-5744 (1993)

4. Laskin, J., Futrell, J.H.: Activation of large ions in FT-ICR mass spectrometry. Mass Spectrom. Rev. 24, 135-167 (2005)

5. Gauthier, J.W., Trautman, T.R., Jacobson, D.B.: Sustained off-resonance irradiation for collision-activated dissociation involving Fourier-transform mass-spectrometry-collision-activated dissociation technique that emulates infrared multiphoton dissociation. Anal. Chim. Acta. 246, 211-225 (1991)

6. Mortensen, D.N., Dearden, D.V.: Influence of charge repulsion on binding strengths: experimental and computational characterization of mixed alkali metal complexes of decamethylcucurbit[5] uril in the gas phase. Chem. Commun. 47, 6081-6083 (2011)

7. Zhang, H., Ferrell, T.A., Asplund, M.C., Dearden, D.V.: Molecular beads on a charged molecular string: $\alpha$, $\omega$-alkyldiammonium complexes of cucurbit[6] uril in the gas phase. Int. J. Mass Spectrom. 265, 187-196 (2007)

8. Mortensen, D.N., Jones, C.A., Dearden, D.V.: Appropriate choice of event length in sustained off-resonance irradiation collision-induced dissociation (SORI-CID) experiments: activated ion collision-induced dissociation. Int. J. Mass Spectrom. 330/332, 241-245 (2012)

9. Yang, F., Voelkel, J., Dearden, D.V.: Collision cross-sectional areas from analysis of fourier transform ion cyclotron resonance linewidth: a new method for characterizing molecular structure. Anal. Chem. 84, 48514857 (2012)

10. Peacock, R.N.: Vacuum Gauges. In: Lafferty, J.M. (ed.) Foundations of Vacuum Science and Technology, pp. 375-446. Wiley, New York (1998)

11. Bayard, R.T., Alpert, D.: Extension of the low pressure range of the ionization gauge. Rev. Sci. Instrum. 21, 571-572 (1950)

12. Penning, F.M.: Ein neues manometer für niedrige gasdrucke, insbesondere zwischen $10^{-3}$ und $10^{-5} \mathrm{~mm}$. Phys. 4, 71-75 (1937)

13. Hobson, J.P., Redhead, P.A.: Operation of an inverted-magnetron gauge in the pressure range $10^{-3}$ to $10^{-12} \mathrm{~mm} \mathrm{Hg}$. Can. J. Phys. 36, 271-288 (1958)

14. Dearden, D.V., Liang, Y., Nicoll, J.B., Kellersberger, K.A.: Study of gas phase molecular recognition using Fourier transform ion cyclotron resonance mass spectrometry (FTICR/MS). J. Mass Spectrom. 36, 989-997 (2001)

15. Lange, W.J., Singleton, J.H., Eriksen, D.P.: Calibration of low pressure penning discharge type gauges. J. Vac. Sci. Technol. 3, 338-344 (1966)

16. Yang, F., Jones, C.A., Dearden, D.V.: Effects of kinetic energy and collision gas on measurement of cross sections by Fourier transform ion cyclotron resonance mass spectrometry. Int. J. Mass Spectrom. (2014) doi:10.1016/j.ijms.2014.07.026

17. Barker, R.A., Ridge, D.P.: Ion-polar neutral momentum transfer collision frequencies: a theoretical approach. J. Chem. Phys. 64, 4411-4416 (1976)

18. Bartmess, J.E., Griffith, S.S.: Tautomerization energetics of benzoannelated toluenes. J. Am. Chem. Soc. 112, 2931-2936 (1990)

19. Phelps, A.V.: Cross-sections and swarm coefficients for nitrogen ions and neutrals in $\mathrm{N}_{2}$ and argon ions and neutrals in Ar for energies from $0.1 \mathrm{eV}$ to 10 keV. J. Phys. Chem. Ref. Data 20, 557-573 (1991)

20. Lanucara, F., Holman, S.W., Gray, C.J., Eyers, C.E.: The power of ion mobility-mass spectrometry for structural characterization and the study of conformational dynamics. Nat. Chem. 6, 281-294 (2014)

21. Lapthorn, C., Pullen, F., Chowdhry, B.Z.: Ion mobility spectrometry-mass spectrometry (IMS-MS) of small molecules: separating and assigning structures to ions. Mass Spectrom. Rev. 32, 43-71 (2013)

22. Wyttenbach, T., Bowers, M.T.: Gas-phase conformations: the ion mobility/ ion chromatography method. Top. Curr. Chem. 225, 207-232 (2003)

23. Williams, J.P., Kipping, M., Vissers, J.P.C.: Traveling wave ion mobility mass spectrometry: proteomic and biopharmaceutical applications. G.I.T. Lab. J., Eur. 16, 36-38 (2012)

24. Thalassinos, K., Scrivens, J.H.: Applications of Traveling Wave Ion Mobility-Mass Spectrometry. In: March, R.E., Todd, J.F.J. (eds.) Applications of Ion Trapping Devices, Practical Aspects of Trapped Ion Mass Spectrometry, pp. 205-236. CRC Press, Boca Raton (2009) 
25. Caravatti, P., Allemann, M.: The 'Infinity Cell': a new trapped-ion cell with radiofrequency covered trapping electrodes for Fourier transform ion cyclotron resonance mass spectrometry. Org. Mass Spectrom. 26, 514-518 (1991)

26. Senko, M.W., Canterbury, J.D., Guan, S., Marshall, A.G.: A highperformance modular data system for Fourier transform ion cyclotron resonance mass spectrometry. Rapid Commun. Mass Spectrom. 10, 1839-1844 (1996)

27. Blakney, G.T., Hendrickson, C.L., Quinn, J.P., Marshall, A.G.: New hardware for ultrahigh resolution and/or data-dependent SWIFT ion isolation in an FT-ICR mass spectrometer. Proceedings of the 56th ASMS Conference on Mass Spectrometry and Allied Topics. Denver, CO, June 1-5 (2008)

28. Jiao, C.Q., Ranatunga, D.R.A., Vaughn, W.E., Freiser, B.S.: A pulsed-leak valve for use with ion trapping mass spectrometers. J. Am. Soc. Mass Spectrom. 7, 118-122 (1996)

29. Marshall, A.G., Hendrickson, C.L., Jackson, G.S.: Fourier transform ion cyclotron resonance mass spectrometry: a primer. Mass Spectrom. Rev. 17, 1-35 (1998)

30. Zubarev, R.A., Makarov, A.: Orbitrap mass spectrometry. Anal. Chem. 85, $5288-5296(2013)$ 